\title{
End-to-End QoS Improvement using IPv6 Header Reduction over MPLS
}

\author{
Imad J. Mohamad, PhD \\ Dept. of Computer Science - College of Science \\ University of Baghdad - Iraq
}

\begin{abstract}
In 802.16 standards, PHS suppresses redundant parts of payload header in MAC service data unit. This paper proposes Label Switched Path-Payload Header Suppression (LSPPHS), in which an MPLS-enabled backbone route compresses packets over an MPLS LSP without compression or decompression cycles at each router. It has two main contributions for MPLS-IPv6 header compression. First, LSPPHS adds a new facility (MPLS/PHS) to the existing MPLS facilities. Second, it provides an analysis of the effect of implementing LSP-PHS on real-time and non-real-time IPv6 traffic in terms of QoS metrics. The implementation results using NS 2.34 show QoS improvement for real-time and nonreal-time traffic.
\end{abstract}

\section{General Terms}

The static fields of IPv6 header represent redundant information (overhead) in data transmission over network. IPv6 Header suppression over MPLS optimizes end-to-end QoS of heterogeneous traffic.

\section{Keywords}

MPLS, IPv6, Header suppression/compression, Real-time and Heterogeneous traffic.

\section{INTRODUCTION}

Real-time applications mainly produce voice and video packets, which represent the main drivers for QoS implementation and traffic engineering mechanisms on the Internet [1]. Real-time applications, such as VoIP, typically produce small payloads, making their packets responsible for the highest relative overheads [2]. "On a VoIP WAN, 300 million calls per day could consume on the order of about 2040 Gbps for headers alone" RFC4247 [3]. For example, a VoIPv6 packet with IPv6/UDP/RTP/Voice encapsulation using GSM 6.0 codec requires 60 bytes for the header and 33 bytes for voice data, or a total of 93 bytes; that is, the header occupies more than half (or 64\%) of the packet size.

MPLS is a routing and forwarding protocol standardized by IETF in 2001. The MPLS domain (cloud) is "a contiguous set of nodes which operate MPLS routing and forwarding and which are also in one Routing or Administrative Domain" [4]. MPLS networks have the capability of minimizing distortions of streams by setting up multiple label switched paths (LSPs), or tunnels, between source and destination to ensure the logical separation between streams [5].

RFC4247 [3] states the requirements for header compression over MPLS and the necessity for header compression in backbone networks. RFC4447 [6] stipulates the rules for setting up pseudo wires using the LDP of MPLS as a signaling protocol. Both RFCs are considered in the proposed LSP-PHS framework. PHS and RoHC over MPLS-LSP is the focus of this paper.
RoHC is a highly robust and efficient header compression scheme for RTP/UDP/IP, UDP/IP, and Encapsulated Security Payload (ESP/IP) headers. It is a standard approach suitable for links with significant error rates and long round-trip time. It has three modes of operation, namely unidirectional, bidirectional optimistic, and bidirectional reliable modes. Header compression with RoHC can be characterized as an interaction between two state machines: a compressor machine and a decompressor machine $[7,8]$.

In the WiMAX PHS scheme, a repetitive portion of the payload headers of the higher layer is suppressed in the MAC service data unit (SDU) by the sending entity, and then restored by the receiving entity. When PHS is enabled at MAC connection, each MAC SDU is prefixed with a Payload Header Suppression Index, which references the Payload Header Suppression Field [7].

RoHC was designed to overcome the implementationlimitations of early designed header compression schemes such as IPHC and ECRTP schemes. These limitations mainly faced in long RTT and high BERs in Wireless, WiMAX and satellite networks. Implementation complexity and restriction from underlying layers are the main limitations of RoHC. For instance, packet length of underlying data link layer is a requirement for RoHC implementation. The advantages and disadvantages of RoHC and PHS are widely investigated in WiMAX networks for mobile to edge-router link. Limited work (if not any) has been done to investigate the performance of RoHC or PHS over MPLS networks.

This paper is organized as follows: background information is provided in Section II. Related work is discussed in Section III. The proposed framework is presented in Section IV. Simulated scenario is discussed in Section V. Section VI concludes this paper.

\section{BACKGROUND}

\subsection{Multiprotocol Label Switch (MPLS)}

Two label edge routers (LERs) or provider edge (PE) routers serve as the ingress and egress of the MPLS domain, and represent the input and output doors of the MPLS cloud (Fig 1). The label switch router (LSR) located one hop before the egress is called penultimate. The penultimate pops the label instead of the egress when this facility is activated. Core LSRs forward the labeled packets without considering their layer 3 headers, and behave as transit LSRs. 


\begin{tabular}{|c|c|c|c|c|c|c|}
\hline \multicolumn{2}{|c|}{ MPLS \& OSI Model } & \multirow{2}{*}{\multicolumn{2}{|c|}{$\begin{array}{l}\text { MPLS } \\
\text { Header }\end{array}$}} & \multirow[b]{2}{*}{$\begin{array}{c}\text { IP } \\
\text { Header }\end{array}$} & \multirow[b]{2}{*}{ Payload } & \multirow[b]{2}{*}{\begin{tabular}{|c} 
Ethernet \\
FCS
\end{tabular}} \\
\hline Layer 5-7 & $\begin{array}{l}\text { Higher layer } \\
\text { applications }\end{array}$ & & & & & \\
\hline Layer 4 & TCP - UDP & & & & & \\
\hline Layer 3 & IPv6 - IPv4 Raw data & & & 1 & & \\
\hline Layer 2.5 & MPLS label & & & $\cos \mathrm{s}$ & TTL & \\
\hline Layer 2 & $\begin{array}{l}\text { PPP - Ethernet - HDLC } \\
\text { - ATM -Frame relay }\end{array}$ & & & & & \\
\hline Layer 1 & Optical -Electrical & & & & & \\
\hline
\end{tabular}

Fig 1: MPLS and OSI model

\subsection{IPv6 Header}

The IPv6 header is divided into eight fields that occupy 40 octets (bytes) determining the fixed length of IPv6 header. Typically, three tuples of IPv6 header, which are the IP source address, IP destination address, and flow label, represent the IPv6 flow signature. At least 120 seconds should pass in order to reuse the same value of flow label for a specific pair of IP source and destination addresses, as specified in RFC 3697 [9]. RFC 3697 has been accepted as the specifications and requirements of IPv6 flow label values. The traffic class field is equivalent to the differentiated services field of IPv4 header, intended for QoS requirements. Generally, header fields of IP protocols can be classified into; Inferred Static, Static-Def, Static Known, Semi-Static, Rarely Changing, or Irregular [10].

\subsection{Size of Static and Dynamic Fields in Profiles}

A simple analysis of the IP profile in RFC3095 showed that 36 of the 40 bytes within the IPv6 header were static fields (SourecIP, DestIP, version, flow label, and next header), 40 of the 48 bytes of the IPv6/UDP profile were static fields (sizes of source and destination ports of UDP were added), and 44 of the 60 bytes of the IPv6/UDP/RTP profile were static information (size of the SSRC static field for RTP was added). Converting these values into percentages (Fig 2) indicates that the percentages of static field sizes to overall packet header size of various ROHC profiles are as follows: 90, 83, and 73\% static information in IPv6, IPv6/UDP, and IPv6/UDP/RTP profiles, respectively. The average of these three ratios yields $82 \%$ represents the static header information of the three sources of data. The high percentage of static fields is an advantage for PHS as compared with the complexity of ROHC.

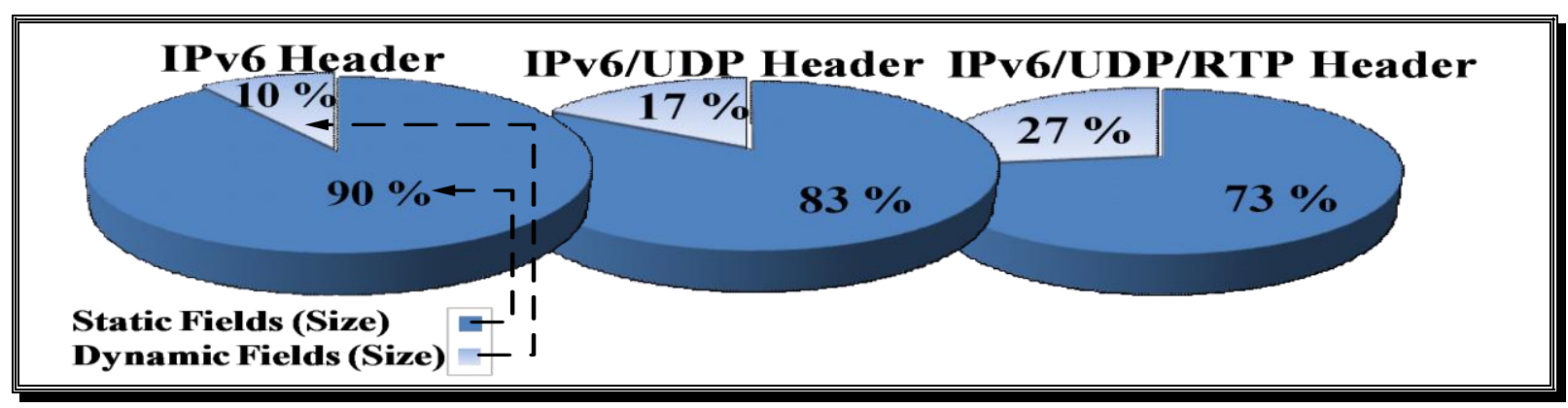

Fig 2: Size of Static and Dynamic Fields in Three Packet-Header Profiles

\section{RELATED WORK}

An enhanced uplink resource allocation algorithm (ertPS+) was evaluated by [11] to maximize VoIP capacity in the mobile WiMAX system. The performance of simulated ertPS+ was evaluated using PHS and RoHC schemes with ITU-T G.729B codec. In their simulation, the assumption was that the RTP/UDP/IP header was compressed to 16 and 2 bytes in PHS and ROHC, respectively. The results showed 23.1 and $31.2 \%$ improvement in VoIP capacity using PHS and RoHC, respectively. However, the paper did not discuss the overhead of RoHC which was equivalent to the difference (8\%) between PHS and RoHC improvements. In addition, the paper focused only on the RTP/UDP/IP profile. Consequently, the $8 \%$ difference is expected to decrease if other RoHC profiles with higher static header fields (such as the IPv6 profile) are tested.

Hardware implementation for WiMAX RoHC was evaluated in Taylor et al.'s paper [12], in which the main workload distributions observed were as follows: $14 \%$ for packet classification, $33 \%$ for packet parsing, 14 to $20 \%$ for CRC computation, and $33 \%$ for header encoding and decoding. Comparatively, the PHS scheme depends on header suppression of static fields with no necessity for encoding/decoding and CRC computation, which were designed functionally for dynamic RoHC fields. Consequently, the last two workloads (14 to 20\% and 33\%) and their storage resources requirements are expected to represent extra RoHC overhead over PHS in terms of space and time complexity.
The performance of RoHC in the global information grid was investigated in [13]. The advantages and disadvantages of RoHC were discussed through a comparison between IPHC and RoHC. The implementation of header compression at the network edges (e.g., MPLS edge routers) was recommended because these routers can possess the necessary processing resources to execute header compression algorithms and consequently utilize the network bandwidth. Furthermore, the decision to implement header compression depended on the tradeoff of node resources, such as processor and memory, for bandwidth savings.

RoHC robustness was discussed and was found unnecessary in [2] for links with low BER or confirmed frame delivery service. The paper highlighted the added $\mathrm{RoHCv} 2$ feature for supporting out-of-order delivery of RoHC packets with further discussion on the less robust or unidirectional mode. The design of backbone networks is expected to address the BER problem.

PHS and RoHC performance for WiMAX was investigated and compared by [14] who found better compression capability for RoHC compared with PHS. In terms of performance evaluation, PHS reduced the RTP/UDP/IPv6 header from 60 to 15 bytes, whereas $\mathrm{RoHC}$ reduced the same header to 4 to 10 bytes on average. The authors also concluded that PHS can perform better when larger static portions of the header found avoids unnecessary computation overheads for dynamic fields. 
An IPv6 integration and coexistence strategy for nextgeneration networks was discussed in [15]. Dual-stack edge routers of MPLS are one of the robust choices for Internet backbone infrastructure. LSP-PHS requires IPv6-aware (or dual stack) edge routers for the MPLS cloud.

\section{PROPOSED METHODOLOGY}

This paper is an extension of the algorithms and experiments in our previous work [16] for further IPv6 QoS investigation. Heterogeneous traffic for real-time and non-real-time IPv6 streams competing for congested bandwidths is tested. This work is also an expansion of the investigation on the effect of LSP-PHS in terms of QoS parameters, such as delay, throughput, and jitter of TCP and UDP stream.

Backbone networks, such as MPLS-enabled backbones, are expected to support high speed, high traffic loads, and low BER compared with an access network. Therefore, PHS is the preferred compression scheme over RoHC due to its simplicity. In a high speed network, the transmission media requires simple yet effective header compression. The header compression mechanism needs to be fast enough to avoid unnecessary delays in feedback and signaling. Although RoHC performs better in terms of robustness, the frequent states transition in RoHC does not suit the MPLS backbone, which has a low bit error rate. LSP-PHS implementation is expected to add less complexity overhead and decrease resource consumption for MPLS edge routers compared to RoHC.

The reader invited to refer to the basic algorithms [16] which were developed to support header suppression and restoration in Ingress and Penultimate LSRs of MPLS. These algorithms extended to study the reaction of TCP traffic to the header suppression of real time traffic or UDP real time traffic. Also more QoS parameters studied compared to our previous work.

In the current paper, the static IPv6 header (36 bytes) is suppressed in tested Scenario. There is also an additional 1 byte that represents the hop limit field that will be suppressed in the implementation of LSP-PHS. The additional byte will bring the number of bytes to a total of 37 that will be suppressed in LSP-PHS. The hop limit field of IPv6 can be suppressed because normal MPLS deals with the ingress packet. This ingress packet copies the hop limit value to the TTL field of the MPLS header at ingress, decremented by one at each hope of an LSP, then copied back to the decremented value (TTL value of the MPLS header) to the hop limit field of IPv6 at penultimate/egress LSR. Consequently, 37 out of 40 bytes in the IPv6 profile are suppressed temporarily between head ends of an MPLS LSP.

Four bytes for LSP-PHS header added to support the proposed methodology as follows: 4 bits for compression states and identification of LSP-PHS frames, 20 bits for Context ID (CID), and 1 byte for PHSI field. For GSM packet size 93 bytes, the net suppression size is $(93-37+4=60)$ bytes which yields to $(60 / 93)$ or $(64.52 \%)$ header compression ratio.

\section{SIMULATION AND RESULTS}

The simulation experiment represented in this section was conducted to check the QoS using LSP-PHS. The topology (Fig. 3) consisted of an edge router, LSR7, which receives its incoming streams from seven routers, LSR0 to LSR6, compresses VoIPv6 packets using the LSP-PHS algorithm, and forwards the compressed packet into LSP towards the egress router or LSR12. The restoration process was implemented in the penultimate or LSR11. In our simulation model, we assumed that differentiated services are not activated for IPv6 packets. LSP-PHS performance was evaluated using the following equations of end-to-end QoS metrics:

- Throughput using Equation (1): DataThroughput $(\mathrm{I})=\frac{\text { No_of_Bits received }}{\text { Second }(\mathrm{I})}$ for $\mathrm{I}=1$ to $\mathrm{N}$ seconds

- Delay using Equation (2): $\operatorname{delay}(i)=\operatorname{recvtime}(i)-\operatorname{sendtime}(i)$

- Jitter (delay variation) using Equation (3): Jitter $=\frac{((\text { recvtime }(j)-\operatorname{sendtime}(j))-(\text { recvtime }(i)-\text { sendtime }(i)))}{(j-i)}, j>i$

- Packets Drop for UDP or retransmitted packets for TCP (counted by summation).

Simulation parameters for tested scenario (Fig. 3) are set as follows: Generated VoIPv6 UDP Flows (0-4) set to GSM 6.0, 33 bytes payload, and 93 bytes as a total packet size using 13 Kbps constant bit rate (CBR). Two TCP flows, FTP/TCP0 and FTP/TCP1, generated using 1000 and 500 bytes respectively. The traffic passes through seven hops that share same LSP path (LSR7 to LSR12). Link-delay set to $10 \mathrm{~ms}$ in all the hops in the topology.

The simulation scenario shows a $64.52 \%$ or $60 / 93$ header compression ratio when LSP-PHS is implemented in the edge routers of the MPLS cloud. The QoS parameters for real-time traffic also improve when both traffic patterns share the same LSP of MPLS. Fig. 4 shows how priority is given to UDP over TCP flows and to small packets over large packets whenever there is insufficient bandwidth during data transmission. Packets drop for the UDP flow of 93-byte packet declines from 72 to 0 or $100 \%$. This is because UDP data will always be prioritized during bandwidth allocation; thus, the packet drops will be reduced. Packet drop for TCP/FTP stream of 500-byte packet size declines from 28 to 12 or $51 \%$, whereas TCP/FTP of 1000-byte packet size declines from 36 to 17 or $53 \%$.

In addition to packet drop, LSP-PHS provides better throughput for TCP and UDP streams, as shown in Fig 5 and 6 , respectively. A change in window size of TCP transmission results in better throughput.

A wider variation of around 6 to $12 \mathrm{Kbps}$ can be observed for UDP data when LSP-PHS is disabled compared with that of the activation of LSP-PHS, which is around 6 to $8 \mathrm{Kbps}$. Decreasing the packet drop of small UDP packets contributes to the increase in throughput for UDP streams.

Fig. 7 shows that the implementation of LSP-PHS diminishes the delay in VoIPv6 streams. It is believed that header suppression reduces traffic congestion in all LSRs; thus, queuing and transmission delays for UDP packets diminish. A minor improvement in jitter is observed for UDP flows (Fig. 8). Table 1 summarizes the results of Scenario.

\section{CONCLUSIONS}

In terms of end-to-end QoS metrics, LSP-PHS implementation using NS2 for the tested Scenarios shows considerable reduction of approximately up to $50 \%$ in UDP maximum delay. Additionally, it eliminates packet drop for real-time traffic (VoiPv6). Bandwidth utilization in MPLS core (links) and savings in resources of LSRs (queues) offered by LSP-PHS to MPLS cloud contributed in the optimization of end-to-end QoS metrics for heterogeneous traffic. 


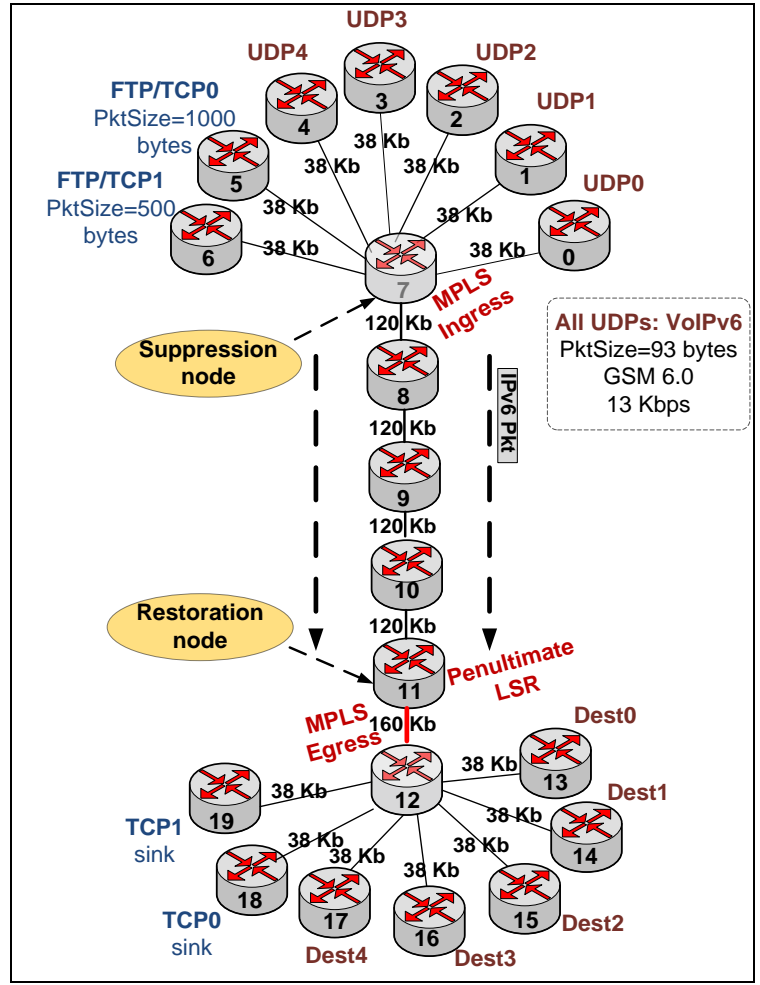

Fig 3: MPLS topology (Scenario)

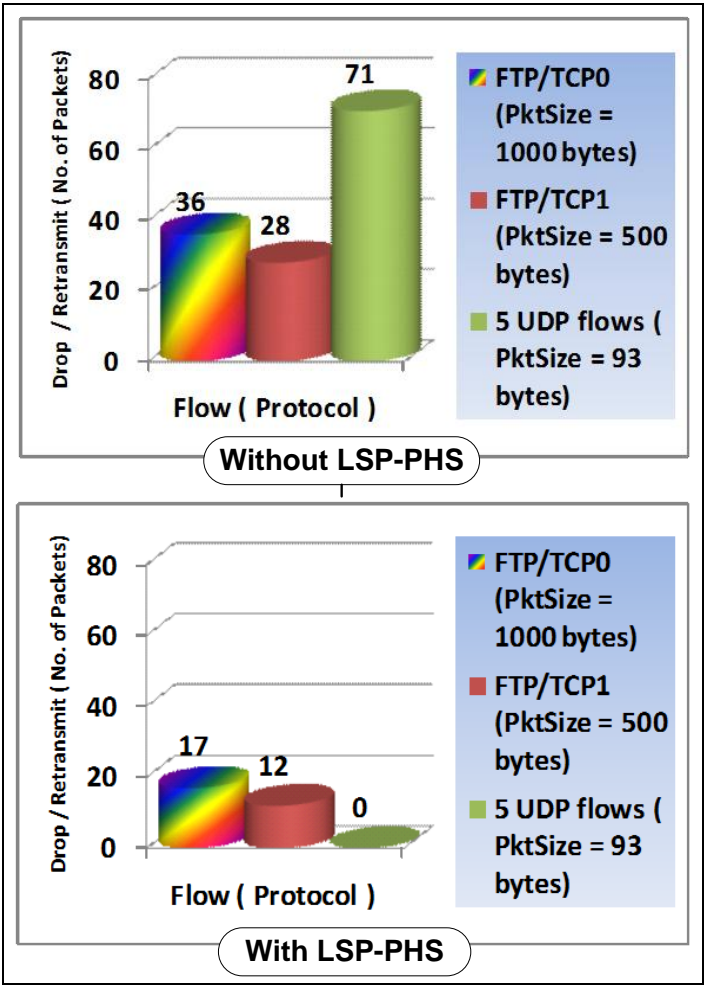

Fig 4: Drop/ Retransmit Statistics

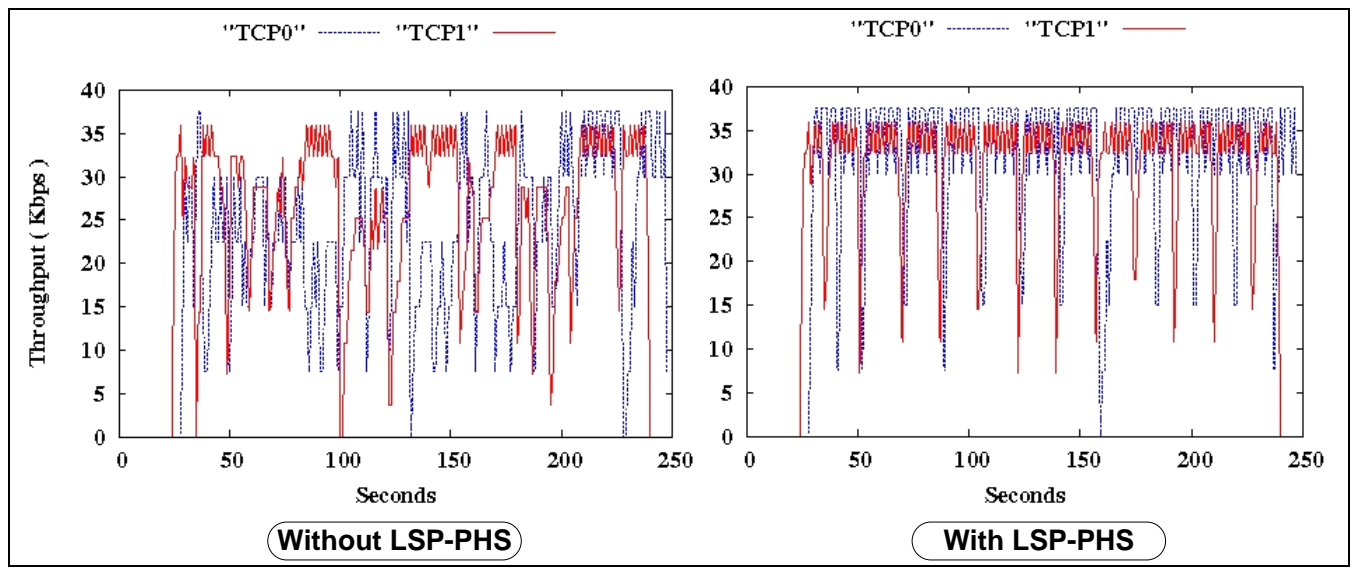

Fig 5: TCP Throughput

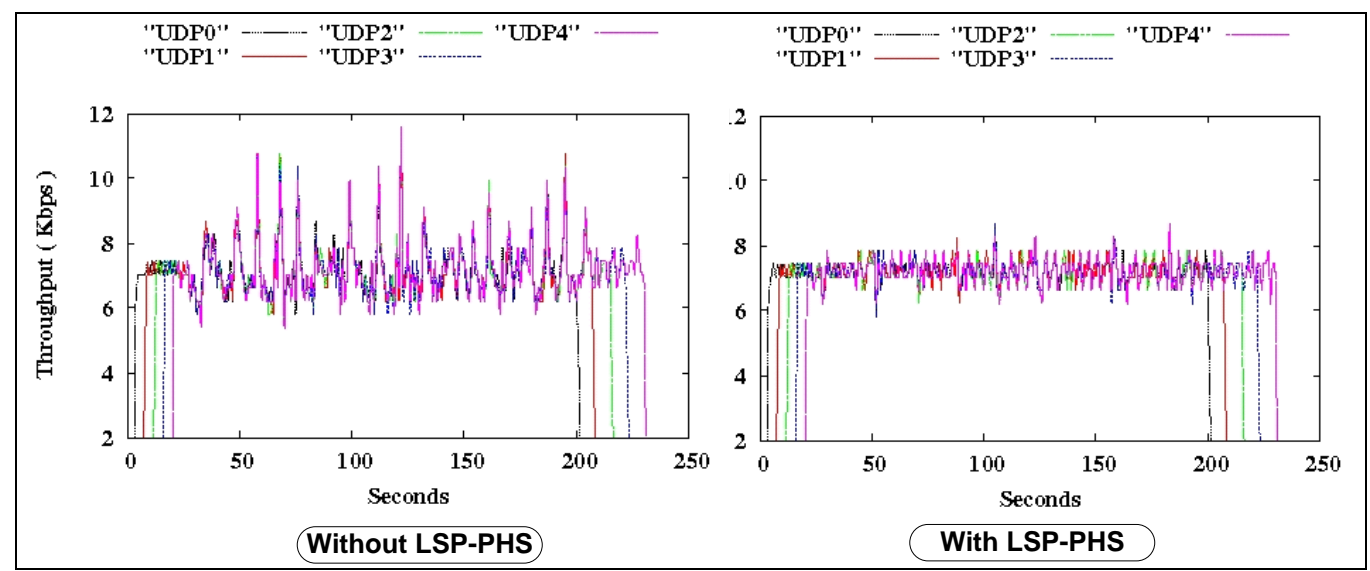

Fig 6: UDP Throughput 


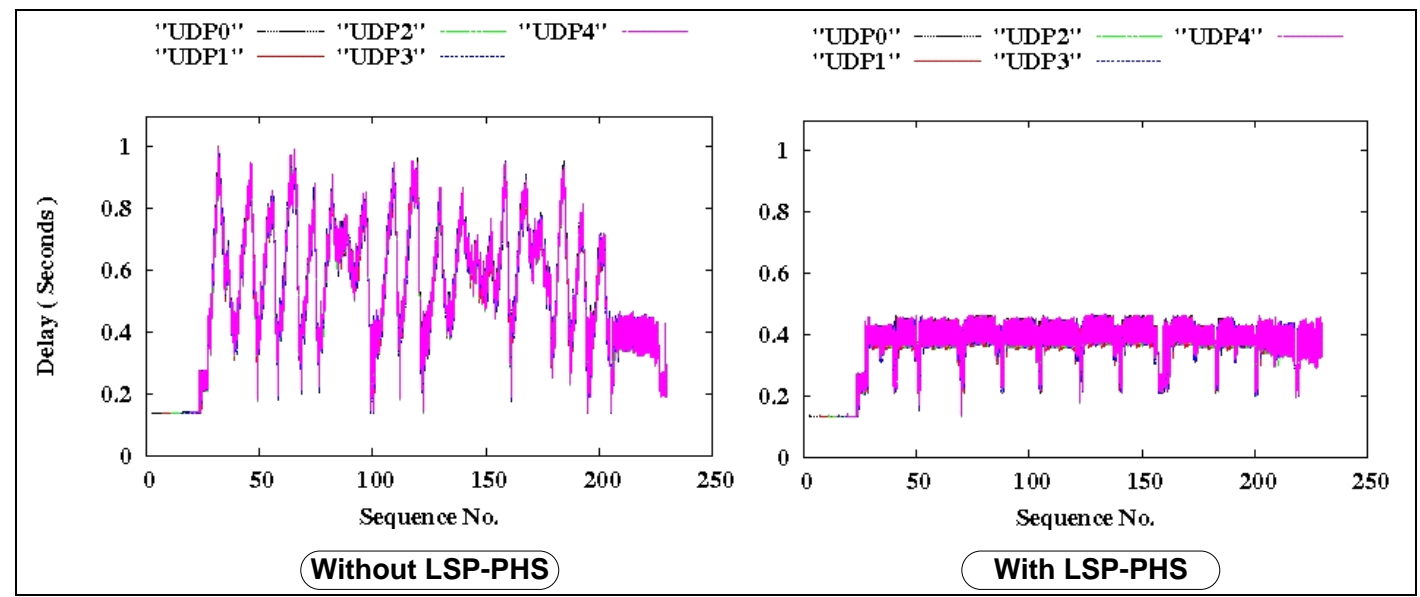

Fig 7: UDP Delay

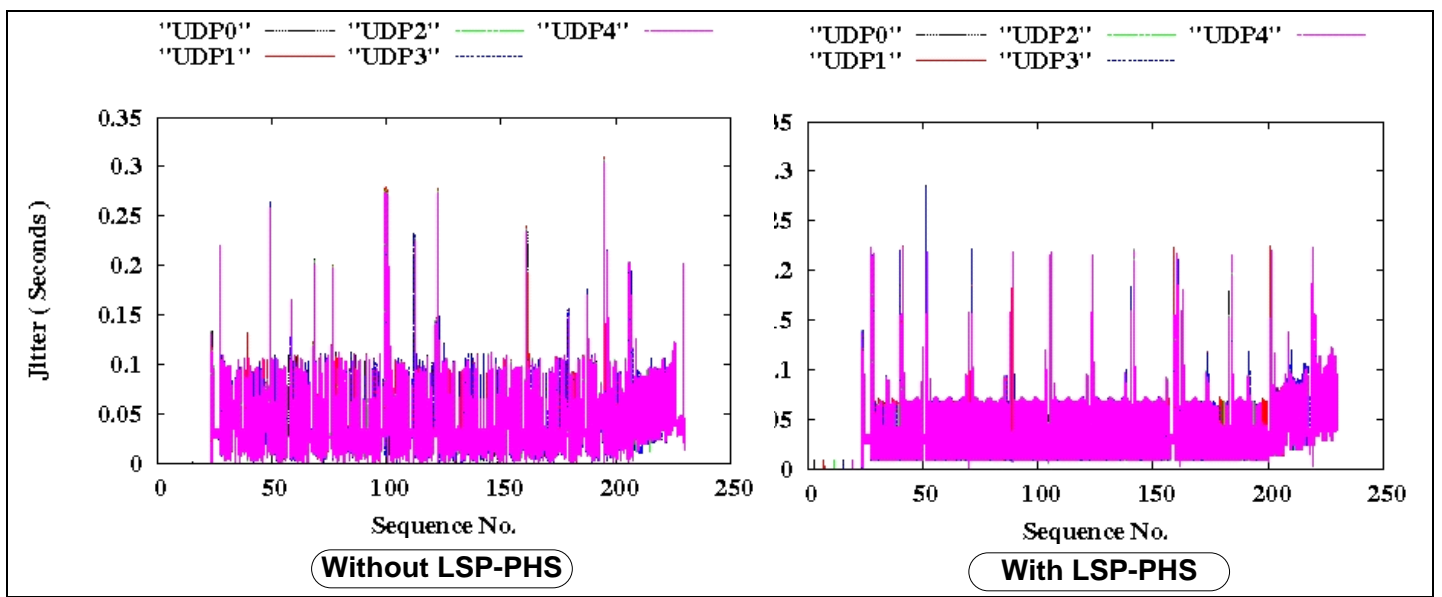

Fig 8: UDP Jitter (Delay variation)

Table 1. Summary for scenario results

\begin{tabular}{|c|c|c|c|c|c|c|}
\hline \multirow{2}{*}{ QoS metrics } & \multicolumn{2}{|c|}{$\begin{array}{c}\text { UDP } \\
(93 \text { bytes)Pkt }\end{array}$} & \multicolumn{2}{|c|}{$\begin{array}{c}\text { FTP/TCP0 } \\
(1000 \text { bytes }) \text { Pkt }\end{array}$} & \multicolumn{2}{|c|}{$\begin{array}{c}\text { FTP/TCP1 } \\
\text { (500 bytes)Pkt }\end{array}$} \\
\hline & $\begin{array}{l}\text { Without } \\
\text { LSP-PHS }\end{array}$ & $\begin{array}{c}\text { With } \\
\text { LSP-PHS }\end{array}$ & $\begin{array}{l}\text { Without } \\
\text { LSP-PHS }\end{array}$ & $\begin{array}{c}\text { With } \\
\text { LSP-PHS }\end{array}$ & $\begin{array}{l}\text { Without } \\
\text { LSP-PHS }\end{array}$ & $\begin{array}{l}\text { With } \\
\text { LSP-PHS }\end{array}$ \\
\hline Throughput & $\begin{array}{l}\text { High variation, } \\
\text { between } 6 \text { to } 12 \\
\text { Kbps }\end{array}$ & $\begin{array}{c}\text { Tuned with Less } \\
\text { variation between } \\
6 \text { to } 8 \mathrm{Kbps}\end{array}$ & $\begin{array}{l}\text { High variation } \\
\text { and most time } \\
\text { under } 30 \mathrm{Kbps}\end{array}$ & $\begin{array}{c}\text { Maximized } \\
\text { and Tuned } \\
\text { between } 30 \text { to } \\
38 \mathrm{Kbps} \\
\end{array}$ & $\begin{array}{l}\text { High variation } \\
\text { and most time } \\
\text { under } 30 \mathrm{Kbps}\end{array}$ & $\begin{array}{c}\text { Maximized } \\
\text { and Tuned } \\
\text { between } 32 \text { to } \\
36 \mathrm{Kbps} \\
\end{array}$ \\
\hline Delay & $\begin{array}{l}\text { high variation } 0.2 \\
\text { to } 1 \mathrm{sec}\end{array}$ & $\begin{array}{c}\text { Reduced to } 50 \% \text {, } \\
\text { between } 0.2 \text { to } 0.5 \\
\text { sec }\end{array}$ & \multicolumn{2}{|c|}{ N/A } & \multicolumn{2}{|c|}{ N/A } \\
\hline Jitter & \multicolumn{2}{|c|}{ Minor improvement } & \multicolumn{2}{|c|}{ N/A } & \multicolumn{2}{|c|}{ N/A } \\
\hline $\begin{array}{l}\text { UDPdrop or } \\
\text { TCPretransmit }\end{array}$ & $72 \mathrm{Pkt}$ & $\begin{array}{l}\text { Reduced to } 0 \mathrm{Pkt} \\
\text { Improved } 100 \%\end{array}$ & $36 \mathrm{Pkt}$ & $\begin{array}{l}17 \mathrm{Pkt}, \\
\text { Improved } \\
53 \%\end{array}$ & $28 \mathrm{Pkt}$ & $\begin{array}{l}12 \mathrm{Pkt}, \\
\text { Improved } \\
51 \%\end{array}$ \\
\hline
\end{tabular}




\section{ACKNOWLEDGMENTS}

Our thanks to the experts who have contributed towards development of the template.

\section{REFERENCES}

[1] A. Meddeb, "Internet QoS: Pieces of the puzzle," Communications Magazine, IEEE, 2010. 48(1): pp. 8694, doi: 10.1109/MCOM.2010.5394035

[2] P. Fortuna, and M. Ricardo, "Header compressed VolP in IEEE 802.11," Wireless Commun., 2009. 16(3): pp. 6975.

[3] J. Ash, J. Hand, and R. Zhang, "Requirements for Header Compression over MPLS," [RFC4247], 2005.

[4] Rosen, E., A. Viswanathan, and R. Callon, "Multiprotocol Label Switching Architecture," [RFC3031], 2001.

[5] S. Srivastava, A.V.D. Liefvoort, and D. Medhi, "Traffic engineering of MPLS backbone networks in the presence of heterogeneous streams," Comput. Netw., 2009. Vol. 53(15): pp. 2688-2702.

[6] L. Martini, et al., "Pseudowire Setup and Maintenance using the Label Distribution Protocol (LDP)," [RFC4447], 2006.

[7] IEEE Std 802.16 $16^{\mathrm{TM}}-2004.2004$.

[8] K. Sandlund, G. Pelletier, and L-E. Jonsson, "The RObust Header Compression (ROHC) Framework,"[RFC5795], 2010.

[9] J. Rajahalme, A. Conta, B. Carpenter, and S. Deering, "IPv6 Flow Label Specification,"[RFC3697], 2004.
[10] G. Pelletier, and K. Sandlund, "RObust Header Compression Version 2 (ROHCv2): Profiles for RTP, UDP, IP, ESP and UDP-Lite," [RFC5225], 2008.

[11] L. Howon, K. Hyu-Dae, and C. Dong-Ho "Smart resource allocation algorithm considering voice activity for VoIP services in mobile-WiMAX system," Wireless Communications, IEEE Transactions on, 2009. Vol. 8(9): pp. 4688-4697.

[12] D. E. Taylor, A. Herkersdorf, A. Döring, and G Dittmann, "Robust header compression (ROHC) in nextgeneration network processors," IEEE/ACM Trans. Netw., 2005. Vol.13(4): pp. 755-768.

[13] E. Ertekin, and C.A. Christou, "Internet protocol header compression, robust header compression, and their applicability in the global information grid," Communications Magazine, IEEE, 2004. Vol. 42(11): pp. 106-116, doi: 10.1109/MCOM.2004.1362553.

[14] L. Nuaymi, N. Bouida, N. Lahbil, and P. Godlewski, "Headers Overhead Estimation, Header Suppression and Header Compression in WiMAX," Proc. of 3rd IEEE International Conference on Wireless and Mobile Computing, Networking and Communications, Oct. 2007. WiMOB 2007, doi 10.1109/WIMOB.2007.38

[15] Tatipamula, M., Grossetete, P., \& Esaki, H. (2004). IPv6 integration and coexistence strategies for next-generation networks. Communications Magazine, IEEE, 42(1), 8896

[16] I.J. Mohamad, T.C. Wan, F.Y. Alzyoud, P. Sumari, "Optimizing MPLS Support for Real Time IPv6-Flows using MPLS-PHS Approach," Proc. IEEE TENCON 2009, Singapore, Nov 23-26, 2009, doi: 10.1109/TENCON.2009.5396094 